CARPATHIAN J. MATH.

Volume 37 (2021), No. 2,

Pages 173 - 184
Online version at https : //www . carpathian. cunbm . utcluj. ro/

Print Edition: ISSN 1584 - 2851; Online Edition: ISSN 1843 - 4401

DOI: https://doi.org/10.37193/CJM.2021.02.03

Dedicated to Prof. Ioan A. Rus on the occasion of his $85^{\text {th }}$ anniversary

\title{
Fixed point theorems for enriched Ćirić-Reich-Rus contractions in Banach spaces and convex metric spaces
}

\author{
VASILE BERINDE ${ }^{1,2}$ and MĂDĂLINA PĂCURAR ${ }^{3}$
}

ABSTRACT. The main aim of this paper is to establish some fixed point theorems for enriched Ćirić-Reich-Rus contractions in Banach spaces and convex metric spaces, respectively.

\section{INTRODUCTION}

Let $(X, d)$ be a metric space. Fifty years ago, in 1971, Ćirić [30], Reich [50] and Rus [52] have established independently a fixed point theorem for mappings $T: X \rightarrow X$ satisfying the following condition:

$$
d(T x, T y) \leq a d(x, y)+b(d(x, T x)+d(y, T y)), \text { for all } x, y \in X,
$$

where $a, b \geq 0$ and $a+2 b<1$.

We remark that, if $b=0$, condition (1.1) reduces to Banach's contraction condition

$$
d(T x, T y) \leq a \cdot d(x, y), \text { for all } x, y \in X,
$$

where $a \in[0,1)$, while for $a=0$ condition (1.1) reduces to Kannan's contraction condition

$$
d(T x, T y) \leq b(d(x, T x)+d(y, T y)), \text { for all } x, y \in X,
$$

where $b \in[0,1 / 2)$.

Therefore, the fixed point results established in [30], [50] and [52], under slightly different forms, are genuine generalizations of Banach's contraction principle [7], [25] and of Kannan's fixed point theorem [38], [39], as shown by the next two examples.

Example 1.1 ([52]). Let $X=[0,1]$ with the usual distance and $T: X \rightarrow X$ given by $T x=\frac{7 x}{20}$, for $0 \leq x \leq \frac{1}{2}$ and $T x=\frac{3 x}{10}$, for $\frac{1}{2}<x \leq 1$. Then $T$ satisfies (1.1) with $a=\frac{1}{10}$ and $b=\frac{4}{9}$ but $T$ does not satisfy neither Banach's contraction condition (1.2) ( $T$ is not continuous) nor Kannan's contraction condition (1.3) (too see that, just take $x=\frac{1}{2}$ and $y=1$ in (1.3) to get the contradiction $14 \leq 2 b \cdot 13<13)$.

Example 1.2 ([50]). Let $X=[0,1]$ with the usual distance and $T: X \rightarrow X$ given by $T x=\frac{x}{3}$, for $0 \leq x<1$ and $T x=\frac{1}{6}$, for $x=1$. Then $T$ satisfies (1.1) with $a=\frac{1}{6}$ and $b=\frac{1}{3}$ but $T$ does not satisfy neither Banach's contraction condition (1.2) ( $T$ is not continuous)

Received: 31.03.2021. In revised form: 20.04.2021. Accepted: 27.04.2021

2010 Mathematics Subject Classification. 47H10, 47H05, 47H09, 47H14, 47H19, 54H25.

Key words and phrases. Banach space, convex metric space, enriched Ćirić-Reich-Rus contraction, fixed point.

Corresponding author: Vasile Berinde; vberinde@cunbm.utcluj.ro 
nor Kannan's contraction condition (1.3) (too see that, just take $x=\frac{1}{3}$ and $y=0$ in (1.3) to get the contradiction $1 \leq 2 b<1$ ).

It is really amazing that, after a half century since the Ćirić-Reich-Rus contractions were introduced, this class of mappings still attracts a remarkable interest amongst researchers working in fixed point theory, see [4], [5], [6], [10], [28] [32]-[37], [40], [41], [44]-[47], [49], [61] etc. From the above list, 13 papers having in their title the syntagm "Ćirić-Reich-Rus" - under various permutations of the names - were published in the last ten years.

On the other hand, in the recent papers [12]-[14], [17]-[19], [22] and [23], the authors used the technique of enriching contractive type mappings in order to generalize, in the setting of a Hilbert space, Banach space or convex metric space, well known and important classes of contractive type mappings from the metric fixed point theory.

Starting from the above mentioned facts, the main aim of this paper is to establish some fixed point theorems for enriched Ćirić-Reich-Rus contractions in Banach spaces and convex metric spaces, respectively.

\section{ENRICHED ĆIRIĆ-REICH-RUS CONTRACTIONS IN BANACH SPACES}

The concept of enriched contraction has been introduced and studied in [17] as a natural generalization of the classical concept of Banach contraction.

Definition 2.1 (Definition 2.1, [17]). Let $(X,\|\cdot\|)$ be a normed linear space. A mapping $T$ : $X \rightarrow X$ is said to be a $(k, a)$-enriched contraction if there exist $k \in[0,+\infty)$ and $a \in[0, k+1)$ such that

$$
\|k(x-y)+T x-T y\| \leq a\|x-y\|, \forall x, y \in X .
$$

Obviously, any Banach contraction satisfies (2.4) with $k=0$. The next theorem is the main result in [17] and represents an effective generalization of Banach's fixed point theorem in the setting of a Banach space. In the following, we shall denote by Fix $(T)$ the set of all fixed points of $T$, that is,

$$
F i x(T)=\{x \in X: T x=x\} .
$$

Theorem 2.1 ([17]). Let $(X,\|\cdot\|)$ be a Banach space and $T: X \rightarrow X a(k, a)$-enriched contraction. Then

(i) Fix $(T)=\{p\}$, for some $p \in X$;

(ii) There exists $\lambda \in(0,1]$ such that the iterative method $\left\{x_{n}\right\}_{n=0}^{\infty}$, given by

$$
x_{n+1}=(1-\lambda) x_{n}+\lambda T x_{n}, n \geq 0,
$$

converges to $p$, for any $x_{0} \in X$;

(iii) The following estimate holds

$$
\left\|x_{n+i-1}-p\right\| \leq \frac{c^{i}}{1-c} \cdot\left\|x_{n}-x_{n-1}\right\|, \quad n=0,1,2, \ldots ; i=1,2, \ldots,
$$

where $c=\frac{a}{k+1}$.

The concept of enriched Kannan contraction has been introduced and studied in [18] as a natural generalization of that of Kannan mapping.

Definition 2.2 (Definition 2.1, [18] ). Let $(X,\|\cdot\|)$ be a linear normed space. A mapping $T: X \rightarrow X$ is said to be a $(k, b)$-enriched Kannan mapping if there exist $k \in[0, \infty)$ and $b \in[0,1 / 2)$ such that

$$
\|k(x-y)+T x-T y\| \leq b(\|x-T x\|+\|y-T y\|), \text { for all } x, y \in X .
$$


Obviously, any Kannan mapping satisfies (2.5) with $k=0$.

The next theorem, the main result in [18], is a genuine generalization of the Kannan fixed point theorem in the setting of a Banach space.

Theorem $2.2([18])$. Let $(X,\|\cdot\|)$ be a Banach space and $T: X \rightarrow X a(k, b)$-enriched Kannan mapping. Then

(i) Fix $(T)=\{p\}$, for some $p \in X$;

(ii) There exists $\lambda \in(0,1]$ such that the iterative method $\left\{x_{n}\right\}_{n=0}^{\infty}$, given by

$$
x_{n+1}=(1-\lambda) x_{n}+\lambda T x_{n}, n \geq 0,
$$

converges to $p$, for any $x_{0} \in X$;

(iii) The following estimate holds

$$
\left\|x_{n+i-1}-p\right\| \leq \frac{\delta^{i}}{1-\delta} \cdot\left\|x_{n}-x_{n-1}\right\|, \quad n=0,1,2, \ldots ; i=1,2, \ldots
$$

where $\delta=\frac{b}{1-b}$.

Our aim in this section is to unify and extend Theorems 2.1 and 2.2 and thus obtain a fixed point theorem for enriched Ćirić-Reich-Rus contractions in Banach spaces.

Definition 2.3. Let $(X,\|\cdot\|)$ be a linear normed space. A mapping $T: X \rightarrow X$ is said to be a $(k, a, b)$-enriched Ćirić-Reich-Rus contraction if there exist $k \in[0, \infty)$ and $a, b \geq 0$, satisfying $a+2 b<1$, such that

$$
\|k(x-y)+T x-T y\| \leq a\|x-y\|+b(\|x-T x\|+\|y-T y\|), \text { for all } x, y \in X .
$$

Obviously, any Ćirić-Reich-Rus contraction satisfies (2.6) with $k=0$.

Also, if $b=0$, then from (2.6) we obtain the contraction condition (2.4) satisfied by an enriched contraction, while, if $a=0$, from (2.6) we obtain the enriched Kannan contraction condition (2.5).

Considering a self-mapping $T$ on $X$, then, for any $\lambda \in(0,1]$, the so-called averaged mapping $T_{\lambda}$ given by

$$
T_{\lambda} x=(1-\lambda) x+\lambda T x, \text { for all } x \in X,
$$

has the property that $F i x\left(T_{\lambda}\right)=F i x(T)$.

The main result of this section is the next theorem.

Theorem 2.3. Let $(X,\|\cdot\|)$ be a Banach space and $T: X \rightarrow X a(k, a, b)$-enriched Ćirić-ReichRus contraction. Then

(i) Fix $(T)=\{p\}$, for some $p \in X$;

(ii) There exists $\lambda \in(0,1]$ such that the iterative method $\left\{x_{n}\right\}_{n=0}^{\infty}$, given by

$$
x_{n+1}=(1-\lambda) x_{n}+\lambda T x_{n}, n \geq 0,
$$

converges to $p$, for any $x_{0} \in X$;

(iii) The following estimate holds

$$
\left\|x_{n+i-1}-p\right\| \leq \frac{\delta^{i}}{1-\delta} \cdot\left\|x_{n}-x_{n-1}\right\|, \quad n=0,1,2, \ldots ; i=1,2, \ldots
$$

where $\delta=\frac{a+b}{1-b}$.

Proof. We work in the case when $k>0$ (the case $k=0$ is immediate) and consider the averaged mapping $T_{\lambda}$ defined by (2.7) for $\lambda=\frac{1}{k+1}<1$. 
In this case we have that $k=1 / \lambda-1$ and thus the contractive condition (2.6) becomes $\left\|\left(\frac{1}{\lambda}-1\right)(x-y)+T x-T y\right\| \leq a\|x-y\|+b(\|x-T x\|+\|y-T y\|)$, for all $x, y \in X$, which can be written equivalently as

$$
\left\|T_{\lambda} x-T_{\lambda} y\right\| \leq a \lambda\|x-y\|+b\left(\left\|x-T_{\lambda} x\right\|+\left\|y-T_{\lambda} y\right\|\right), \text { for all } x, y \in X
$$

and, because $a \lambda \leq a$, this implies that

$$
\left\|T_{\lambda} x-T_{\lambda} y\right\| \leq a\|x-y\|+b\left(\left\|x-T_{\lambda} x\right\|+\left\|y-T_{\lambda} y\right\|\right), \text { for all } x, y \in X,
$$

which means that $T_{\lambda}$ is a Ćirić-Reich-Rus contraction mapping.

By using triangle inequality in (2.10), we obtain that $T_{\lambda}$ satisfies

$$
\left\|T_{\lambda} x-T_{\lambda} y\right\| \leq \delta \cdot\|x-y\|+2 \delta \cdot\left\|y-T_{\lambda} x\right\|, \text { for all } x, y \in X
$$

where $\delta=\frac{a+b}{1-b}<1$.

Consider the iterative process $\left\{x_{n}\right\}_{n=0}^{\infty}$ defined by (2.8), which is in fact the Picard iteration associated to $T_{\lambda}$, that is,

$$
x_{n+1}=T_{\lambda} x_{n}, n \geq 0 .
$$

and take $x=x_{n-1}$ and $y=x_{n}$ in (2.11) to get

$$
\left\|x_{n+1}-x_{n}\right\| \leq \delta\left\|x_{n}-x_{n-1}\right\|, n \geq 1 .
$$

By (2.13) one obtains routinely the following two estimates

$$
\left\|x_{n+m}-x_{n}\right\| \leq \delta^{n} \cdot \frac{1-\delta^{m}}{1-\delta} \cdot\left\|x_{1}-x_{0}\right\|, n \geq 0, m \geq 1
$$

and

$$
\left\|x_{n+m}-x_{n}\right\| \leq \delta \cdot \frac{1-\delta^{m}}{1-\delta} \cdot\left\|x_{n}-x_{n-1}\right\|, n \geq 1, m \geq 1 .
$$

Now, by (2.14) it follows that $\left\{x_{n}\right\}_{n=0}^{\infty}$ is a Cauchy sequence and hence it is convergent in the Banach space $(X,\|\cdot\|)$. Let us denote

$$
p=\lim _{n \rightarrow \infty} x_{n}
$$

We first prove that $p$ is a fixed point of $T_{\lambda}$. We have

$$
\left\|p-T_{\lambda} p\right\| \leq\left\|p-x_{n+1}\right\|+\left\|x_{n+1}-T_{\lambda} p\right\|=\left\|x_{n+1}-p\right\|+\left\|T_{\lambda} x_{n}-T_{\lambda} p\right\| .
$$

By (2.11) we deduce that

$$
\left\|T_{\lambda} x_{n}-T_{\lambda} p\right\| \leq \delta\left\|x_{n}-p\right\|+2 \delta\left\|p-x_{n+1}\right\|,
$$

and therefore, by (2.17) we obtain

$$
\left\|p-T_{\lambda} p\right\| \leq(2 \delta+1)\left\|x_{n+1}-p\right\|+\delta\left\|x_{n}-p\right\|, n \geq 0 .
$$

Now, by letting $n \rightarrow \infty$ in (2.18) we get $\left\|p-T_{\lambda} p\right\|=0$, that is, $p=T_{\lambda} p$.

So, $p \in$ Fix $\left(T_{\lambda}\right)$.

Now, in order to prove that $p$ is the unique fixed point of $T_{\lambda}$, we note that by $(2.10)$, similarly to the way we have obtained (2.11), one obtains

$$
\left\|T_{\lambda} x-T_{\lambda} y\right\| \leq \delta \cdot\|x-y\|+2 \delta \cdot\left\|x-T_{\lambda} x\right\|, \text { for all } x, y \in X
$$

where $\delta=\frac{a+b}{1-b}$. 
Assume, that $q \neq p$ is another fixed point of $T_{\lambda}$. Then, by (2.19) with $x=p$ and $y=q$ it follows

$$
0<\|p-q\| \leq \delta\|p-q\|<\|p-q\|,
$$

a contradiction. Hence Fix $\left(T_{\lambda}\right)=\{p\}$ and since Fix $(T)=F i x\left(T_{\lambda}\right),(i)$ is proven.

Conclusion $(i i)$ follows by (2.16).

To prove (iii), we let $m \rightarrow \infty$ in (2.14) and (2.15) to get

$$
\left\|x_{n}-p\right\| \leq \frac{\delta^{n}}{1-\delta} \cdot\left\|x_{1}-x_{0}\right\|, n \geq 1
$$

and

$$
\left\|x_{n}-p\right\| \leq \frac{\delta}{1-\delta} \cdot\left\|x_{n}-x_{n-1}\right\|, n \geq 1,
$$

respectively, and then we merge (2.20) and (2.21) to get the unifying error estimate (2.9).

Remark 2.1. Theorem 2.3 includes as particular cases Theorem 2.1 and Theorem 2.2, which are obtained from Theorem 2.3 for $b=0$ and $a=0$, respectively.

A more general fixed point result can be obtained by allowing the coefficients $a$ and $b$ in the contraction condition (2.6) to depend on $x$ and $y$, like in Theorem 2.5 of Ćirić [30].

Definition 2.4. Let $(X,\|\cdot\|)$ be a linear normed space. A mapping $T: X \rightarrow X$ is said to be a generalized $(k, a, b)$-enriched Ćirić-Reich-Rus contraction if, for every $x, y \in X$, there exist $k \in[0, \infty)$ and the non-negative functions $a, b: X^{2} \rightarrow[0, \infty)$ satisfying

$$
\sup _{x, y \in X}(a(x, y)+2 b(x, y))=\theta<1
$$

such that, for all $x, y \in X$,

$$
\|k(x-y)+T x-T y\| \leq a(x, y)\|x-y\|+b(x, y)(\|x-T x\|+\|y-T y\|) .
$$

The following theorem is a generalization of Theorem 2.3. Its proof follows the same pattern like that of Theorem 2.3 and is omitted.

Theorem 2.4. Let $(X,\|\cdot\|)$ be a Banach space and $T: X \rightarrow X$ a generalized $(k, a, b)$-enriched Cirić-Reich-Rus contraction. Then

(i) Fix $(T)=\{p\}$, for some $p \in X$;

(ii) There exists $\lambda \in(0,1]$ such that the iterative method $\left\{x_{n}\right\}_{n=0}^{\infty}$, given by

$$
x_{n+1}=(1-\lambda) x_{n}+\lambda T x_{n}, n \geq 0,
$$

converges to $p$, for any $x_{0} \in X$;

(iii) The following estimate holds

$$
\left\|x_{n+i-1}-p\right\| \leq \frac{\theta^{i}}{1-\theta} \cdot\left\|x_{n}-x_{n-1}\right\|, \quad n=0,1,2, \ldots ; i=1,2, \ldots
$$

Similarly to the proof of Theorem 2.3 one can prove a local variant of Theorem 2.4:

Theorem 2.5. Let $(X,\|\cdot\|)$ be a Banach space and $\bar{x} \in X$. Denote

$$
B=B(\bar{x}, r)=\{x \in X:\|x-\bar{x}\| \leq r\}, r>0
$$

and let $T: B \rightarrow X$ be a generalized ( $k, a, b)$-enriched Ćirić-Reich-Rus contraction which satisfies the condition

$$
\|\bar{x}-T \bar{x}\| \leq(1-\theta) r
$$

Then

(i) $T$ has a unique fixed point $p \in B$; 
(ii) There exists $\lambda \in(0,1]$ such that the iterative method $\left\{x_{n}\right\}_{n=0}^{\infty}$, given by

$$
x_{n+1}=(1-\lambda) x_{n}+\lambda T x_{n}, n \geq 0,
$$

converges to $p$, for any $x_{0} \in X$;

(iii) The following estimate holds

$$
\left\|x_{n+i-1}-p\right\| \leq \frac{\theta^{i}}{1-\theta} \cdot\left\|x_{n}-x_{n-1}\right\|, \quad n=0,1,2, \ldots ; i=1,2, \ldots
$$

where $\theta=\sup _{x, y \in X}(a(x, y)+2 b(x, y))$.

We remark that, due to the particular form of the contractive conditions (2.4), (2.5), (2.6) and the corresponding ones in [12], [13], [14], [17], [18], [19], [22], which involve explicitly the linearity of the space $X$, all those results were established in the case of a Banach space or of a Hilbert space.

On the other hand, the basic fixed point theorems established in literature for PicardBanach contractions [25], Kannan mappings [38, 39], Chatterjea mappings [26], ĆirićReich-Rus contractions [30], [50], [52] etc. are usually stated in the setting of a metric space.

Therefore, the main aim of the next section is to extend the results presented in the current section for enriched Ćirić-Reich-Rus contractions to the more general case of a convex metric space in the sense of Takahashi.

\section{ENRICHED ĆIRIĆ-REICH-RUS CONTRACTIONS IN CONVEX METRIC SPACES}

In 1970, Takahashi [60] introduced a structure of convexity outside linear spaces which turned out to be very useful in fixed point theory.

Definition $3.5([60])$. Let $(X, d)$ be a metric space. A continuous function $W: X \times X \times$ $[0,1] \rightarrow X$ is said to be a convex structure on $X$ if, for all $x, y \in X$ and any $\lambda \in[0,1]$,

$$
d(u, W(x, y ; \lambda)) \leq \lambda d(u, x)+(1-\lambda) d(u, y) \text {, for any } u \in X .
$$

A metric space $(X, d)$ endowed with a convex structure $W$ is called a Takahashi convex metric space and is usually denoted by $(X, d, W)$.

Obviously, any linear normed space and each of its convex subsets are convex metric spaces, with the natural convex structure

$$
W(x, y ; \lambda)=\lambda x+(1-\lambda) y, x, y \in X, \lambda \in[0,1],
$$

but the reverse is not valid: there are various examples of convex metric spaces which cannot be embedded in any Banach space (see [60], Example 1; [1], Examples 1 and 2; [2], [29], [42] etc.).

The next lemmas present some fundamental properties of a convex metric space in the sense of Definition 3.5 (see [60, 2] for more details and [23] for their proofs).

Lemma 3.1. Let $(X, d, W)$ be a convex metric space. For all $x, y \in X$ and any $\lambda \in[0,1]$,

$$
d(x, y)=d(x, W(x, y ; \lambda))+d(W(x, y ; \lambda), y) .
$$

Lemma 3.2. Let $(X, d, W)$ be a convex metric space. For all $x, y \in X$ and any $\lambda \in[0,1]$, we have

$$
d(x, W(x, y ; \lambda))=(1-\lambda) d(x, y) \text { and } d(W(x, y ; \lambda), y)=\lambda d(x, y) .
$$

Lemma 3.3. Let $(X, d, W)$ be a convex metric space. For each $x, y \in X$ and $\lambda, \lambda_{1}, \lambda_{2} \in[0,1]$, we have the following:

(i) $W(x, x ; \lambda)=x ; \quad W(x, y ; 0)=y$ and $W(x, y ; 1)=x$;

(ii) $\left|\lambda_{1}-\lambda_{2}\right| d(x, y) \leq d\left(W\left(x, y ; \lambda_{1}\right), W\left(x, y ; \lambda_{2}\right)\right)$. 
Let $(X, d, W)$ be a convex metric space and $T: X \rightarrow X$ be a self mapping.

The next lemma is a partial extension of a result given as a Corollary to Theorem 5 in [24], from the setting of Banach spaces to that of convex metric spaces.

Lemma 3.4. Let $(X, d, W)$ be a convex metric space and $T: X \rightarrow X$ be a self mapping. Define the mapping $T_{\lambda}: X \rightarrow X$ by

$$
T_{\lambda} x=W(x, T x ; \lambda), x \in X .
$$

Then, for any $\lambda \in[0,1)$,

$$
\operatorname{Fix}(T)=F i x\left(T_{\lambda}\right) .
$$

Definition 3.6. Let $(X, d, W)$ be a convex metric space. A mapping $T: X \rightarrow X$ is said to be a $(k, a, b)$-enriched Ćirić-Reich-Rus contraction if there exist $k \in[0, \infty)$ and $a, b \geq 0$ satisfying $a+2 b<1$ such that for all $x, y \in X$

(3.28) $d(W(x, T x ; \lambda), W(y, T y ; \lambda)) \leq a d(x, y)+b(d(x, W(x, T x ; \lambda))+d(y, W(y, T y ; \lambda)))$.

Example 3.3. Let $(X,\|\cdot\|)$ be a linear normed space and $W(x, y ; \lambda)$ the natural convex structure on $X$, that is,

$$
W(x, y ; \lambda)=\lambda x+(1-\lambda) y, x, y \in X, \lambda \in[0,1] .
$$

Then, a $(k, a, b)$-enriched Ćirić-Reich-Rus contraction $T: X \rightarrow X$ in the sense of Definition 3.6 is a $(k, a, b)$-enriched Ćirić-Reich-Rus contraction in the sense of Definition 2.3.

The next theorem is an extension of Theorem 2.3 from Banach spaces to convex metric spaces.

Theorem 3.6. Let $(X, d, W)$ be a complete convex metric space and let $T: X \rightarrow X$ be $a(k, a, b)$ enriched Ćirić-Reich-Rus contraction. Then,

(i) Fix $(T)=\{p\}$, for some $p \in X$.

(ii) The sequence $\left\{x_{n}\right\}_{n=0}^{\infty}$ obtained from the iterative process

$$
x_{n+1}=W\left(x_{n}, T x_{n} ; \lambda\right), n \geq 0,
$$

converges to $p$, for any $x_{0} \in X$.

(iii) The following estimate holds

$$
d\left(x_{n+i-1}, p\right) \leq \frac{\delta^{i}}{1-\delta} \cdot d\left(x_{n}, x_{n-1}\right) \quad n=1,2, \ldots ; i=1,2, \ldots
$$

where $\delta=\frac{a+b}{1-b}$.

Proof. By the enriched contractive condition (3.28), we have that the mapping $T_{\lambda}: X \rightarrow X$ defined by (3.26) satisfies

$$
d\left(T_{\lambda} x, T_{\lambda} y\right) \leq a \cdot d(x, y)+b\left(d\left(x, T_{\lambda} x\right)+d\left(y, T_{\lambda} y\right)\right), \text { for all } x, y \in X,
$$

that is, $T_{\lambda}$ is a Ćirić-Reich-Rus contraction. We note that the Picard iteration associated to $T_{\lambda}$ is actually the Krasnoselskij iterative process $\left\{x_{n}\right\}_{n=0}^{\infty}$ associated to $T$ and defined by (3.29), i.e.,

$$
x_{n+1}=T_{\lambda} x_{n}, n \geq 0 .
$$

By using triangle inequality from (3.31) we get

$$
d\left(T_{\lambda} x, T_{\lambda} y\right) \leq \delta \cdot d(x, y)+2 \delta d\left(y, T_{\lambda} x\right), \text { for all } x, y \in X,
$$

and

$$
d\left(T_{\lambda} x, T_{\lambda} y\right) \leq \delta \cdot d(x, y)+2 \delta d\left(x, T_{\lambda} x\right), \text { for all } x, y \in X
$$


where $\delta=\frac{a+b}{1-b}<1$, which shows that $T_{\lambda}$ is an almost contraction, see [8], [21] for more details.

Now, we take $x=x_{n}$ and $y=x_{n-1}$ in (3.33) to get

$$
d\left(x_{n+1}, x_{n}\right) \leq \delta \cdot d\left(x_{n}, x_{n-1}\right), n \geq 1,
$$

which inductively implies

$$
d\left(x_{n+1}, x_{n}\right) \leq \delta^{n} \cdot d\left(x_{1}, x_{0}\right), n \geq 1 .
$$

By (3.35) one obtains routinely the following two estimates

$$
d\left(x_{n+m}, x_{n}\right) \leq \delta^{n} \cdot \frac{1-\delta^{m}}{1-\delta} \cdot d\left(x_{1}, x_{0}\right), n \geq 0, m \geq 1
$$

and

$$
d\left(x_{n+m}, x_{n}\right) \leq \delta \cdot \frac{1-\delta^{m}}{1-\delta} \cdot d\left(x_{n}, x_{n-1}\right), n \geq 1, m \geq 1
$$

Now, by (3.37) it follows that $\left\{x_{n}\right\}_{n=0}^{\infty}$ is a Cauchy sequence and hence it is convergent in the complete convex metric space $(X, d)$. Let us denote

$$
p=\lim _{n \rightarrow \infty} x_{n}
$$

We first prove that $p$ is a fixed point of $T_{\lambda}$. We have

$$
\begin{gathered}
d\left(p, T_{\lambda} p\right) \leq d\left(p, x_{n+1}\right)+d\left(x_{n+1}, T_{\lambda} p\right)=d\left(x_{n+1}, p\right)+d\left(T_{\lambda} x_{n}, T_{\lambda} p\right) \\
\leq(2 \delta+1) d\left(x_{n+1}, p\right)+\delta d\left(x_{n}, p\right)
\end{gathered}
$$

and by letting $n \rightarrow \infty$ we get $d\left(p, T_{\lambda} p\right)=0$, that is, $p=T_{\lambda} p$.

So, $p \in F i x\left(T_{\lambda}\right)$.

Now, in order to prove that $p$ is the unique fixed point of $T_{\lambda}$, just assume that $q \neq p$ is another fixed point of $T_{\lambda}$. Then, $d(p, q)>0$ and from (3.34) with $x=p$ and $y=q$ we get

$$
0<d(p, q) \leq \delta d(p, q)<d(p, q),
$$

a contradiction. Hence $\operatorname{Fix}\left(T_{\lambda}\right)=\{p\}$ and since Fix $(T)=F i x\left(T_{\lambda}\right),(i)$ is proven.

Conclusion (ii) follows by (3.39).

To prove (iii), we let $m \rightarrow \infty$ in (3.37) and (3.38) to get

$$
d\left(x_{n}, p\right) \leq \frac{\delta^{n}}{1-\delta} \cdot d\left(x_{1}, x_{0}\right), n \geq 1
$$

and

$$
d\left(x_{n}, p\right) \leq \frac{\delta}{1-\delta} \cdot d\left(x_{n}, x_{n-1}\right), n \geq 1,
$$

respectively, and then by merging (3.40) and (3.41) we obtain the unifying error estimate (3.30).

The remaining case $k=0$ is similar to $k>0$ with the only difference that in this case $\lambda=1$ and hence, by Lemma 3.3,

$$
W(x, T x, 0)=T x ; W(y, T y, 0)=T y
$$

and so the contraction condition (3.28) reduces to (1.1), for which we run the proof in the well known manner. Note that Krasnoselskij iteration (3.29) reduces in this case to the simple Picard iteration

$$
x_{n+1}=T x_{n}, n \geq 0 .
$$

Remark 3.2. By Theorem 3.6 we obtain in particular the first main result in [23]. 
Corollary 3.1 ([23], Theorem 1$)$. Let $(X, d, W)$ be a complete convex metric space and let $T$ : $X \rightarrow X$ be a $(\lambda, c)$-enriched contraction. Then,

(i) Fix $(T)=\{p\}$, for some $p \in X$.

(ii) The sequence $\left\{x_{n}\right\}_{n=0}^{\infty}$ obtained by the iterative process

$$
x_{n+1}=W\left(x_{n}, T x_{n} ; \lambda\right), n \geq 0,
$$

converges to $p$, for any $x_{0} \in X$.

(iii) The following estimate holds

$$
d\left(x_{n+i-1}, p\right) \leq \frac{c^{i}}{1-c} \cdot d\left(x_{n}, x_{n-1}\right) \quad n=1,2, \ldots ; i=1,2, \ldots
$$

Proof. We apply Theorem 3.6 for the case $b=0$ in condition (3.28).

The following result is an extension of Theorem 2.2 in [18], from Banach spaces to convex metric spaces.

Corollary 3.2. Let $(X, d, W)$ be a complete convex metric space and let $T: X \rightarrow X$ be $a(k, b)$ enriched Kannan contraction, that is, a mapping for which there exist $k \in[0, \infty)$ and $b \in\left(0, \frac{1}{2}\right)$ such that

$$
d\left(T_{\lambda} x, T_{\lambda} y\right) \leq b(d(x, W(x, T x ; \lambda))+d(y, W(y, T y ; \lambda))), \text { for all } x, y \in X
$$

Then,

(i) Fix $(T)=\{p\}$, for some $p \in X$.

(ii) The sequence $\left\{x_{n}\right\}_{n=0}^{\infty}$ obtained by the iterative process

$$
x_{n+1}=W\left(x_{n}, T x_{n} ; \lambda\right), n \geq 0,
$$

converges to $p$, for any $x_{0} \in X$.

(iii) The following estimate holds

$$
d\left(x_{n+i-1}, p\right) \leq \frac{c^{i}}{1-c} \cdot d\left(x_{n}, x_{n-1}\right) \quad n=1,2, \ldots ; i=1,2, \ldots
$$

where $c=2 b$.

Proof. We apply Theorem 3.6 for the case in which we have $a=0$ in condition (3.28).

We end the paper by stating a fixed point result that extends Theorem 2.4 from Banach spaces to convex metric spaces. We need the following concept.

Definition 3.7. Let $(X, d, W)$ be a convex metric space. A mapping $T: X \rightarrow X$ is said to be a generalized $(k, a, b)$-enriched Ćirić-Reich-Rus contraction if, for every $x, y \in X$ there exist $k \in[0, \infty)$ and the non-negative functions $a, b: X^{2} \rightarrow[0, \infty)$ satisfying

$$
\sup _{x, y \in X}(a(x, y)+2 b(x, y))=\theta<1
$$

such that for all $x, y \in X$

$d(W(x, T x ; \lambda), W(y, T y ; \lambda)) \leq a(x, y) d(x, y)+b(x, y)(d(x, W(x, T x ; \lambda))+d(y, W(y, T y ; \lambda)))$.

Obviously, any $(k, a, b)$-enriched Ćirić-Reich-Rus contraction is a generalized $(k, a, b)$ enriched Ćirić-Reich-Rus contraction but the reverse is not valid in general.

Therefore, the following result is a generalization of Theorem 2.3. 
Theorem 3.7. Let $(X, d, W)$ be a complete convex metric space and $T: X \rightarrow X$ a generalized $(k, a, b)$-enriched Ćirić-Reich-Rus contraction. Then

(i) Fix $(T)=\{p\}$, for some $p \in X$;

(ii) The sequence $\left\{x_{n}\right\}_{n=0}^{\infty}$ obtained from the iterative process

$$
x_{n+1}=W\left(x_{n}, T x_{n} ; \lambda\right), n \geq 0,
$$

converges to $p$, for any $x_{0} \in X$.

(iii) The following estimate holds

$$
d\left(x_{n+i-1}, p\right) \leq \frac{\theta^{i}}{1-\theta} \cdot d\left(x_{n}, x_{n-1}\right) \quad n=1,2, \ldots ; i=1,2, \ldots
$$

where $\theta=\sup _{x, y \in X}(a(x, y)+2 b(x, y))$.

\section{CONCLUSIONS}

1. Based on the technique of enriching contractive type mappings $T$ by means of the averaged operator $T_{\lambda}$, we introduced the concept of enriched Ćirić-Reich-Rus contraction in Banach spaces and convex metric spaces.

2. We established fixed point theorems for enriched Ćirić-Reich-Rus contractions and generalized enriched Ćirić-Reich-Rus contractions in both settings (Banach spaces and convex metric spaces).

3. The obtained results are important generalizations of the corresponding results for enriched contractions and enriched Kannan mappings in Banach spaces and also for enriched contractions in convex metric spaces, respectively. For other possible directions of research we refer to [3], [8], [9], [11], [16], [20], [21], [31], [43], [48], [53]-[59], [62],...

4. Most of the authors of the recent papers that bear in their title the names of the three mathematicians Ćirić, Reich and Rus to which we owe the important class of contractions we studied in this paper are using the alphabetical order "Ćirić-Reich-Rus". However, if we look to the submission dates, we can see that the paper by Rus [52] has been submitted in February 1971, the one by Ćirić [30] in June 1971, while Reich's paper [50] has no submission information.

So, if we would use the chronological order for the concept we were dealing with, it should be called "Rus-Ćirić-Reich", but we adopted the alphabetical order which is usually accepted. Other combinations we can find: "Reich-Rus-Ćirić" ([32], [45]), "Rus-ReichĆirić" ([37], [40], [41]) etc.

Acknowledgments. The paper is a modest but sincere homage to our esteemed PhD supervisor, Professor Emeritus Ioan A. Rus, Honorary member of Romanian Academy, who guided both authors in their very first and later steps in the wonderful world of Fixed Point Theory.

\section{REFERENCES}

[1] Abbas, M., Common fixed point results with applications in convex metric space, Fasc. Math., 39 (2008), 5-15

[2] Agarwal, R. P., O'Regan, D. and Sahu, D. R., Fixed point theory for Lipschitzian-type mappings with applications in Topological Fixed Point Theory and Its Applications., 6. Springer, New York, 2009

[3] Alghamdi, M., Berinde, V. and Shahzad, N., Fixed point of multivalued nonself almost contractions, J. Appl. Math., 2013, Art. ID 621614, 6 pp.

[4] Altun, I. and Taşdemir, A., On best proximity points of interpolative proximal contractions Quaes. Math., DOI: $10.2989 / 16073606.2020 .1785576$

[5] Aydi, H., Chen, Chi-Ming and Karapinar, E., Interpolative Ciric-Reich-Rus Type Contractions via the Branciari Distance, Mathematics 7 (2019), No. 1, Article Number: 84 
[6] Aydi, H., Karapinar, E. and Róldan Lopez de Hierro, A. F., w-Interpolative Ciric-Reich-Rus-Type Contractions, Mathematics 7 (2019), No. 1, Article Number: 57

[7] Banach, S., Sur les opérations dans les ensembles abstraits et leurs applications aux équations intégrales, Fundam. Math.,3 (1922), 133-181

[8] Berinde, V., Approximating fixed points of weak contractions using the Picard iteration, Nonlinear Anal. Forum 9 (2004), Mo. 1, 43-53

[9] Berinde, V., Iterative Approximation of Fixed Points, Springer, 2007

[10] Berinde, V., General constructive fixed point theorems for Ćirić-type almost contractions in metric spaces, Carpathian J. Math., 24 (2008), No. 2, 10-19

[11] Berinde, V., Approximating fixed points of implicit almost contractions, Hacettepe J. Math. Stat., 41 (2012), No. $1,93-102$

[12] Berinde, V., Weak and strong convergence theorems for the Krasnoselskij iterative algorithm in the class of enriched strictly pseudocontractive operators, An. Univ. Vest Timiş. Ser. Mat.-Inform., 56 (2018), No. 2, 13-27

[13] Berinde, V., Approximating fixed points of enriched nonexpansive mappings by Krasnoselskij iteration in Hilbert spaces, Carpathian J. Math., 35 (2019), No. 3, 293-304

[14] Berinde, V., Approximating fixed points of enriched nonexpansive mappings in Banach spaces by using a retractiondisplacement condition Carpathian J. Math., 36 (2020), No. 1, 27-34

[15] Berinde, V. and Păcurar, M., Coupled fixed point theorems for generalized symmetric Meir-Keeler contractions in ordered metric spaces, Fixed Point Theory Appl., 2012, 2012:115, 11 pp.

[16] Berinde, V. and Păcurar, M., Fixed point theorems for nonself single-valued almost contractions, Fixed Point Theory, 14 (2013), No. 2, 301-311

[17] Berinde, V. and Păcurar, M., Approximating fixed points of enriched contractions in Banach spaces, J. Fixed Point Theory Appl., 22 (2020), No. 2, Paper 38, 10 pp.

[18] Berinde, V. and Păcurar, M., Kannan's fixed point approximation for solving split feasibility and variational inequality problems, J. Comput. Appl. Math., 386 (2021), 113217, 9 pp.

[19] Berinde, V. and Păcurar, M., Fixed point theorems for Chatterjea type mappings in Banach spaces, J. Fixed Point Theory Appl., (submitted)

[20] Berinde, V. and Rus, I. A., Asymptotic regularity, fixed points and successive approximations, Filomat 34 (2020), No. 3, 965-981

[21] Berinde, V. and Păcurar, M., Iterative approximation of fixed points of single-valued almost contractions in Fixed Point Theory and Graph Theory, 29-97, Elsevier/Academic Press, Amsterdam, 2016

[22] Berinde, V. and Păcurar, M., Krasnoselskij-type algorithms for variational inequality problems and fixed point problems in Banach spaces, arXiv:2103.10289

[23] Berinde, V. and Păcurar, M., Existence and Approximation of Fixed Points of Enriched Contractions and Enriched $\varphi$-Contractions, Symmetry-Basel, 13 (2021), No. 3, Article no.: 498, https://doi.org/10.3390/sym13030498.

[24] Browder, F. E. and Petryshyn, W. V., Construction of fixed points of nonlinear mappings in Hilbert space, J. Math. Anal. Appl., 20 (1967), 197-228

[25] Caccioppoli, R., Un teorema generale sull'esistenza di elementi uniti in una transformazione funzionale, Rend. Accad. Lincei., 11 (1930), 794-799

[26] Chatterjea, S. K., Fixed-point theorems, C. R. Acad. Bulgare Sci., 25 (1972), 727-730

[27] Chen, L., Yang, N., Zhao, Y. and Ma, Z., Fixed point theorems for set-valued G-contractions in a graphical convex metric space with applications, J. Fixed Point Theory Appl., 22 (2020), No. 4, Paper No. 88, 23 pp.

[28] Chifu, C. and Petruşel, G., Generalized contractions in metric spaces endowed with a graph, Fixed Point Theory Appl., 2012, 2012:161, 9 pp.

[29] Choban, M. M., About convex structures on metric spaces Carpathian J. Math. 37 (2021) (in press)

[30] Ćirić, L. B., Generalized contractions and fixed-point theorems, Publ. Inst. Math. (Beograd) (N.S.), 12(26) (1971), No. 26, 19-26

[31] Ćirić, L. B., A generalization of Banach's contraction principle, Proc. Am. Math. Soc., 45 (1974), 267-273

[32] Debnath, P., Mitrović, Z. D. and Radenović, S., Interpolative Hardy-Rogers and Reich-Rus-Ćirić type contractions in b-metric spaces and rectangular b-metric spaces, Mat. Vesnik, 72 (2020), No. 4, 368-374

[33] Debnath, P. and de La Sen, M., Fixed-Points of Interpolative Ciric-Reich-Rus-Type Contractions in b-Metric Spaces, Symmetry-Basel, 12 (2020), No. 1, Article Number: 12

[34] Debnath, P. and de La Sen, M., Set-Valued Interpolative Hardy-Rogers and Set-Valued Reich-Rus-Ciric-Type Contractions in b-Metric Spaces, Mathematics, 7 (2019), No. 9, Article Number: 849

[35] Errai, Y., Marhrani, El M. and Aamri, M., Fixed Points of g-Interpolative Ciric-Reich-Rus-Type Contractions in b-Metric Spaces, Axioms 9 (2020), No. 4, Article Number: 132

[36] Gautam, P., Mishra, V. N. and Negi, K., Common fixed point theorems for cyclic Ćirić-Reich-Rus contraction mappings in quasi-partial b-metric space, Ann. Fuzzy Math. Inform., 20 (2020), No. 2, 149-156 
[37] Gautam, P., Sanchez Ruiz, L. M. and Verma, S., Fixed Point of Interpolative Rus-Reich-Ciric Contraction Mapping on Rectangular Quasi-Partial b-Metric Space, Symmetry-Basel, 13 (2021), No. 1, Article Number: 32

[38] Kannan, R., Some results on fixed points, Bull. Calcutta Math. Soc., 60 (1968), 71-76

[39] Kannan, R., Some results on fixed points. II, Am. Math. Monthly, 76 (1969), 405-408

[40] Karapinar, E., Agarwal, R. P. and Aydi, H., Interpolative Reich-Rus-Ciric Type Contractions on Partial Metric Spaces, Mathematics, 6 (2018), No. 11, Article Number: 256

[41] Karapinar, E. and Agarwal, R. P., Interpolative Rus-Reich-Ćirić type contractions via simulation functions, An. Ştiinţ. Univ. "Ovidius" Constanţa Ser. Mat., 27 (2019), No. 3, 137-152

[42] Khan, S. H. and Abbas, M., Common fixed point results with applications in convex metric spaces, J. Concr. Appl. Math., 10 (2012), 65-76

[43] Meszaros, J., A comparison of various definitions of contractive type mappings, Bull. Calcutta Math. Soc., 84 (1992), No. 2, 167-194

[44] Mirzaee, S. and Gordji, M. E., $(G, \psi)$-Ciric-Reich-Rus contraction on metric space endowed with a graph, Int. J. Nonlinear Anal. Appl., 11 (2020), No. 1, 191-197

[45] Mishra, V. N., Sánchez Ruiz, L. M., Gautam, P. and Verma, S., Interpolative Reich-Rus-Ćirić and Hardy-Rogers Contraction on Quasi-Partial b-Metric Space and Related Fixed Point Results, Mathematics, 8 (2020), No. 9, Article Number: 1598

[46] Mishra, L. N., Mishra, V. N., Gautam, P. and Negi, K., Fixed point theorems for Cyclic-Ćirić-Reich-Rus contraction mapping in quasi-partial b-metric spaces, Sci. Publ. State Univ. Novi Pazar Ser. A: Appl. Math. Inform. And Mech., 12 (2020), No. 1, 47-56

[47] Mohammadi, B., Parvaneh, V. and Aydi, H., On extended interpolative Ćirić-Reich-Rus type F-contractions and an application, J. Inequal. Appl., 2019, Paper No. 290, 11 pp.

[48] Păcurar, M., Iterative methods for fixed point approximation, Editura Risoprint, Cluj-Napoca, 2009

[49] Petric, M. A., Some remarks concerning Ćirić-Reich-Rus operators, Creat. Math. Inform., 18 (2009), No. 2, 188-193

[50] Reich, S., Some remarks concerning contraction mappings, Canad. Math. Bull., 14 (1971), 121-124

[51] Rhoades, B. E., A comparison of various definitions of contractive mappings, Trans. Amer. Math. Soc., 226 (1977), 257-290

[52] Rus, I. A., Some fixed point theorems in metric spaces, Rend. Istit. Mat. Univ. Trieste, 3 (1971), 169-172, (1972)

[53] Rus, I. A., Principles and Applications of the Fixed Point Theory (in Romanian), Editura Dacia, Cluj-Napoca, 1979

[54] Rus, I. A.. Metrical Fixed Point Theorems, Univ. of Cluj-Napoca, 1979

[55] Rus, I. A., Generalized contractions, Seminar on Fixed Point Theory, 3 (1983) 1-130

[56] Rus, I. A.,Generalized Contractions and Applications, Cluj Univ. Press, Cluj-Napoca, 2001

[57] Rus, I. A., Picard operators and applications, Sci. Math. Jpn., 58 (2003), No. 1, 191-219

[58] Rus, I. A., Heuristic introduction to weakly Picard operator theory, Creat. Math. Inform., 23 (2014), No. 2, 243-252

[59] Rus, I. A., Petruşel, A. and Petruşel, G., Fixed Point Theory, Cluj University Press, Cluj-Napoca, 2008

[60] Takahashi, W., A convexity in metric space and nonexpansive mappings. I., Kōdai Math. Sem. Rep., 22 (1970), 142-149

[61] Zakany, M., New classes of local almost contractions, Acta Univ. Sapientiae Math., 10 (2018), No. 2, 378-394

[62] Zamfirescu, T., Fix point theorems in metric spaces, Arch. Math. (Basel), 23 (1972), 292-298

${ }^{1}$ Department of Mathematics and Computer SCience

TECHNICAL UNIVERSITY OF CLUJ-NAPOCA

North University Centre at Baia MARE

VICTORIEI 76, RO-430122 BAIA MARE, ROMANIA

E-mail address: vberinde@cunbm.utcluj.ro

${ }^{2}$ ACAdemy OF Romanian ScIENTISTS

E-mail address: vasile.berindelgmail.com

3 Department of Economics and Bussiness Administration in GERMAn LANGUAGE

FACULTY OF ECONOMICS AND BUSSINESS ADMINISTRATION

BABEŞ-BOLYAI UNIVERSITY OF ClUJ-NAPOCA

T. Mihali 58-60, 400591 Cluj-NaPOCA, ROMANiA

E-mail address: madalina.pacurareecon.ubbcluj.ro 